Meta

Journal des traducteurs

Translators' Journal

\title{
Faut-il être juriste ou traducteur pour traduire le droit ?
}

\section{Judith Lavoie}

Volume 48, numéro 3, septembre 2003

Traduction et enseignement

Translation and teaching

URI : https://id.erudit.org/iderudit/007599ar

DOI : https://doi.org/10.7202/007599ar

Aller au sommaire du numéro

Éditeur(s)

Les Presses de l'Université de Montréal

ISSN

0026-0452 (imprimé)

1492-1421 (numérique)

Découvrir la revue

\section{Citer cet article}

Lavoie, J. (2003). Faut-il être juriste ou traducteur pour traduire le droit ? Meta, 48(3), 393-401. https://doi.org/10.7202/007599ar

\section{Résumé de l'article}

Selon une croyance qui semble fortement ancrée, on suppose que les juristes sont " naturellement " plus aptes à traduire des textes juridiques que les non-juristes. Le présent article s’attache, d'une part, à démontrer le contraire en faisant une synthèse des différents travaux ayant porté sur la formation des traducteurs juridiques et, d'autre part, à présenter les grandes lignes de l'enseignement de cette discipline dans le cadre d'un cours avancé portant sur le droit des sociétés et les valeurs mobilières, des domaines en pleine expansion sur le marché de la traduction.
Ce document est protégé par la loi sur le droit d'auteur. L'utilisation des services d'Érudit (y compris la reproduction) est assujettie à sa politique d'utilisation que vous pouvez consulter en ligne.

https://apropos.erudit.org/fr/usagers/politique-dutilisation/ 


\title{
Faut-il être juriste ou traducteur pour traduire le droit?
}

\author{
JUDITH LAVOIE \\ Université de Montréal, Montréal, Canada \\ judith.lavoie@umontreal.ca
}

\begin{abstract}
RÉSUMÉ
Selon une croyance qui semble fortement ancrée, on suppose que les juristes sont «naturellement» plus aptes à traduire des textes juridiques que les non-juristes. Le présent article s'attache, d'une part, à démontrer le contraire en faisant une synthèse des différents travaux ayant porté sur la formation des traducteurs juridiques et, d'autre part, à présenter les grandes lignes de l'enseignement de cette discipline dans le cadre d'un cours avancé portant sur le droit des sociétés et les valeurs mobilières, des domaines en pleine expansion sur le marché de la traduction.
\end{abstract}

\section{ABSTRACT}

According to a strong belief, jurists are said to be automatically capable of translating legal documents. This paper demonstrates the contrary by proposing an overview of the literature published on legal translation training, and explores a few ways of teaching legal translation in an advanced course focusing on corporate law and securities, two mainstream fields in professional practice today.

\section{MOTS-CLÉS/KEYWORDS}

traduction juridique, enseignement de la traduction juridique, droit des sociétés, valeurs mobilières

\section{Introduction}

Cette question - Faut-il être juriste ou traducteur pour traduire le droit? - peut être posée dans diverses spécialités. On peut en effet se demander s'il est préférable d'avoir une formation en pharmacologie pour traduire des textes de nature pharmaceutique, s'il faut être comptable pour traduire des bilans financiers ou mécanicien pour traduire un manuel de mécanique. Mais le raisonnement n'est-il pas poussé trop loin? S'attend-on vraiment à ce qu'un traducteur ou une traductrice dans le domaine de la mécanique automobile soit aussi capable d'effectuer l'équilibrage des roues ou de réparer les freins d'une voiture? Bien sûr que non, alors pourquoi les traducteurs et les traductrices qui traduisent des textes à teneur juridique devraientils sinon avoir une double formation (traduction et droit), au moins être juristes? Et comment ce présupposé alimente-t-il l'enseignement même de la traduction juridique au niveau universitaire? Voilà les questions auxquelles tentera de répondre le présent article, qui se subdivise en deux parties, la première proposant une synthèse des travaux ayant porté sur la formation des traducteurs juridiques, la seconde présentant ma propre pratique pédagogique, qui se veut une sorte de prolongement des recherches et réflexions qui ont porté sur le sujet. 


\section{Quelle formation pour les futurs traducteurs juridiques?}

Parmi les auteurs qui se sont intéressés à l'enseignement de la traduction juridique, un certain nombre s'est posé la question de savoir s'il faut être juriste ou traducteur pour traduire le droit. La plupart d'entre eux choisissent le juste milieu, alléguant que la double formation est la plus indiquée dans le cas qui nous intéresse. David G. Reed, un chercheur qui a travaillé à l'élaboration du vocabulaire de la Common Law en français, est de cet avis, mais semble tout de même croire que la formation en droit devrait primer la formation en traduction:

Les traducteurs de textes juridiques ne sont que trop rarement des juristes et, quand ils le sont, ils sont par trop éloignés des problèmes linguistiques que pose le texte original. Ces traducteurs ignorent trop souvent les nombreux problèmes d'ordre linguistique que présente le droit du Québec, et sont du même fait ignorants des différents concepts couverts par les mêmes termes (1979: 95).

Gabrielle Smart, membre de l'Association des anciens élèves de l'ESIT, semble partager cette vision des choses, précisant en effet que:

De toute façon, il est difficile de traduire un document juridique sans connaître un minimum de droit dans les deux langues concernées, tout comme il est difficile pour un juriste de bien traduire un texte juridique sans une maitrise parfaite de la langue d'arrivée, afin de rendre toutes les nuances du sens si possible à l'identique, car le droit est toujours une affaire d'interprétation des mots (1999: 1).

Pour ces deux auteurs, la double formation semble la plus adéquate.

Michel Sparer, un jurilinguiste qui a enseigné la traduction juridique à l'université pendant plus de vingt ans, penche quant à lui pour la formation en traduction: «Lorsqu'il s'agit de traduire, la clef de la qualité est avant tout l'aptitude à la traduction. En effet, une bonne traduction ne s'improvise pas et il n'est pas évident qu'il suffirait d'être juriste ou psychologue bilingues pour pouvoir faire de la traduction au niveau professionnel dans ces domaines» (2002: 275). Le professeur et chercheur en traduction juridique, Jean-Claude Gémar, s'est lui aussi prononcé sur cette question de la formation du traducteur juridique, notant que l'idée selon laquelle:

$[\mathrm{U}] \mathrm{n}$ juriste est la personne tout indiquée pour traduire des textes juridiques est universellement répandue. Nous prétendons, quant à nous, qu'un juriste qui ne serait pas formé à la traduction, à ses méthodes, à ses contraintes n'est pas plus qualifié pour traduire qu'une secrétaire d'entreprise, un comptable, un architecte, un médecin ou un plombier. Or, particulièrement au Canada, l'on considère qu'un avocat (ou une personne détenant un diplôme de droit), dans la mesure où il possède quelques rudiments de langue seconde ou troisième, est habilité ex officio à traduire des textes de nature juridique (1988: 306).

Donc, pour Gémar et Sparer, il ne suffit pas d'être avocat pour traduire dans le domaine du droit. Je partage leur avis pour deux raisons. En 1987, la revue L'Actualité terminologique consacrait un numéro à la traduction juridique. Roda P. Roberts y signait alors un article intitulé: «Legal Translator and Legal Interpreter Training in Canada» dans lequel elle décrivait un nouveau programme de traduction juridique de deuxième cycle créé à l'Université d'Ottawa. Le programme était uniquement ouvert à des étudiants qui avaient une formation en droit ou une formation en traduction. On souhaitait de cette façon tabler sur les connaissances antérieures de ces 
étudiants. Pour être admis, il fallait aussi réussir un examen d'entrée obligatoire qui testait les étudiants sur leurs connaissances linguistiques.

Or, un an après la création du programme, Roberts tirait déjà certaines conclusions extrêmement intéressantes pour ce qui touche l'enseignement de la traduction juridique, dont les deux suivantes: "Despite the prevailing belief that translators have problems grasping complicated legal concepts, translation graduates in the programme have done exceedingly well in the basic law courses offered to them by the Faculty of Law. [...] Over half of the law graduates who apply for admission fail the entrance exam because of inadequate language skills» (1987: 9). Ces conclusions viennent appuyer l'affirmation de Gémar et celle de Sparer selon lesquelles il ne suffit pas d'être juriste pour traduire des textes juridiques.

D’autre part, Gémar affirmait plus haut que cette idée voulant que les juristes soient plus aptes à traduire des textes juridiques que les non-juristes est une idée universellement répandue. Cette idée universelle vient probablement du fait que le droit est considéré comme un domaine de mots et d'interprétation. On tient donc pour acquis que les juristes savent écrire, et qu'ils écrivent bien, parce que le droit est centré sur les mots, et surtout sur le pouvoir des mots. C'est en effet la position de Reed, qui note que: "Contrarily to many specialized fields that can rely on formulas, be they mathematical or linguistic, as in weather forecasts, [t] he law is a profession of words [...]. That entails that one's understanding of the law will depend in great part on one's knowledge of the language the law is written into. The tools and objects of the law are language » (1993: 84). Si les outils des juristes sont des mots, ces juristes seraient d'office compétents pour faire de la traduction dans le domaine. Une conclusion qui peut sembler rapide, mais que certains se permettent de tirer sans autre forme de procès. Dans un article électronique consacré à la formation des traducteurs juridiques, Jacques Pelage, un enseignant à l'ESIT, affirmait en effet ceci: "On a coutume de dire que le langage est le seul instrument du droit. Tout juriste est donc, au même titre que le traducteur, un spécialiste du langage» (1999: 1). Toutefois, cette position n'est pas celle de Sparer, qui observe que:

La traduction, en droit comme ailleurs, doit être assise sur des compétences particulières en matière linguistique comme en matière de communication. Un texte, juridique ou non, est avant tout un instrument de communication. Il n'est pas si clair que des juristes ou des médecins par exemple aient toujours les aptitudes à la communication qui garantiraient aux lecteurs et aux lectrices un texte d'arrivée compréhensible (2002: 275).

En plus de ne pas nécessairement posséder ce que Sparer appelle des «aptitudes à la communication », de nombreux juristes perpétuent, de façon consciente ou non, des anglicismes qui ont depuis longtemps été mis au jour par des traducteurs, des linguistes et des lexicographes. Pensons par exemple à l'emploi du terme français corporation et ses dérivés, corporatif, incorporation, incorporer, s'incorporer. Bien que le nom de la loi fédérale ait été changé (à deux reprises) pour devenir la Loi canadienne sur les sociétés par actions (elle avait eu pour titre Loi sur les sociétés commerciales canadiennes, et avant cela, Loi sur les corporations commerciales canadiennes), on retrouve le terme corporation et ses dérivés employés de façon fautive dans différents manuels et dans des ouvrages de doctrine importants, comme La compagnie au Québec de Maurice Martel et Paul Martel. Or, bien que ces deux auteurs admettent que le terme de corporation a été délaissé et remplacé par celui de personne morale, 
jugé plus français (1997: 3), ils continuent d'employer les dérivés de corporation dans un sens erroné. On retrouve donc dans leur ouvrage les termes: droit corporatif, calqué de corporate law, au lieu de droit des sociétés; forme corporative, qu'on pourrait remplacer par société tout court; incorporer, à la place de constituer en société; loi corporative au lieu de loi sur les sociétés; et voile corporatif, calqué de l'anglais corporate veil, dont la forme française correcte reste encore à inventer. Nicole Lacasse, dans son ouvrage Droit de l'entreprise, utilise à peu de choses près la même terminologie que Martel et Martel. Dans leur Initiation au droit des affaires, Jean-Pierre Archambault et Marc-André Roy optent pour le terme de compagnie (et non corporation), mais ne dédaignent pas employer les termes entreprise incorporée, entreprise non incorporée et droit corporatif, à l'occasion. Ainsi, malgré le fait que le nom de la loi fédérale ait été changé et que presque tous les dictionnaires de droit s'entendent pour dire que le terme de corporation au sens de société par actions ou de personne morale est à éviter (c'est le cas notamment de Jean-Claude Gémar et Vo Ho-Thuy, qui consacrent plus de deux pages à l'entrée corporation dans leur ouvrage Difficultés $d u$ langage du droit au Canada; et de Wallace Schwab qui relèvent les termes corporation, corporate, incorporation et incorporate), plusieurs juristes continuent de l'employer.

D'autres termes sont calqués abusivement de l'anglais, comme statutaire (de statute, en français loi), ou juridiction (emprunté à l'anglais jurisdiction). Le premier devrait être remplacé par législatif (droit statutaire céderait sa place à droit législatif, et disposition statutaire à disposition législative), le second, quant à lui, pourrait se rendre par autorité législative, territoire, pouvoir ou compétence, selon le contexte.

Ces diverses formes fautives utilisées par certains juristes montrent que des compétences linguistiques sont nécessaires à la pratique de la traduction juridique. Toutefois, il est clair que l'étudiant ou l'étudiante formé-e à la traduction juridique devra, comme tout traducteur professionnel, connaitre les bases du domaine dans lequel il ou elle compte exercer sa profession; en d'autres termes, le fait d'avoir des compétences en traduction n'exempte pas le futur traducteur de détenir des connaissances en droit, l'un n'excluant pas l'autre. En effet, selon Geneviève Mareschal: «La traduction d'un texte spécialisé comporte [...] deux dimensions essentielles: d'une part, l'objet du texte ou son contenu et, d'autre part, la langue du texte ou sa forme » (1988: 258). Monique C. Cormier ajoute un autre élément à ce binôme, des aptitudes à «la recherche documentaire et terminologique» (1990: 676). Enfin, dans un article consacré à l'initiation à la traduction économique - ce qui rejoint mon propos - Jean Delisle propose en quelque sorte une synthèse de ce qui vient d'être décrit, à savoir les

[C]inq objectifs d'apprentissage suivants: 1 . Assimiler les notions fondamentales de l'économie. 2. Assimiler la terminologie de base de l'économie. 3. Assimiler la phraséologie propre aux textes économiques. 4. Apprendre à se documenter sur le sujet. 5. Développer l'aptitude à traduire des textes économiques (1988: 205).

Sans reprendre chacun de ces objectifs dans cet ordre, je tente tout de même de les intégrer à mon approche pédagogique de manière à atteindre cette double visée, notionnelle et linguistique, qui reprend les cinq objectifs précédents, soit 1 et 4 pour l'apprentissage des notions, et 2, 3 et 5 pour le développement des compétences linguistiques. 


\section{Pratique pédagogique de la traduction juridique}

Il convient de préciser d'entrée de jeu que je ne prétends pas inventer une nouvelle méthode d'enseignement de la traduction juridique - les chercheurs cités plus haut ayant largement contribué à le faire -, je souhaite plutôt proposer une conception pédagogique axée à la fois sur les recherches précédentes et sur les besoins actuels des futurs traducteurs et traductrices juridiques.

Une part considérable du marché de la traduction juridique est occupée aujourd'hui par le domaine des valeurs mobilières et du droit des sociétés: prospectus, notice annuelle, bilan financier, avis et circulaires de toutes sortes étant le lot des traducteurs. J'ai donc fait le choix d'orienter certains de mes cours - les cours avancés - vers ce domaine de spécialité, et d'opter, dans le cas d'un cours de base, pour une présentation générale du système juridique canadien et québécois. Il existe, au Département de linguistique et de traduction de l'Université de Montréal, trois cours avancés en traduction juridique; le premier constitue la suite logique du cours d'introduction et se donne dans le cadre du baccalauréat, les deux autres sont offerts à des étudiants de deuxième cycle, l'un au programme de DESS (Diplôme d'études supérieures spécialisées), l'autre, à la maitrise.

L'enseignement se divise concrètement en deux parties: une partie magistrale, où sont abordées des notions de droit précises; et une partie pratique, où sont corrigés ensemble des exercices distribués au cours précédent (cette partie inclut également les travaux pratiques, i.e. les TP). Les cours sont divisés de cette façon afin de répondre à la double visée de l'enseignement de la traduction spécialisée: notionnelle et linguistique. Il faut toutefois préciser que des notions sont également apprises dans le cadre des exercices, et des remarques touchant la terminologie juridique bilingue s'insèrent également à l'enseignement magistral, chaque partie n'étant pas étanche à l'autre. En outre, une telle approche permet à l'étudiant de plonger dès les premières semaines de cours dans le bain de la traduction, car, comme on l'a noté plus haut, il faut d'abord être traducteur pour traduire des textes juridiques.

Pour ce qui concerne la transmission des concepts, je privilégie l'enseignement magistral. Il est toutefois généralement admis que ce type d'enseignement comporte ses lacunes. Les étudiants seraient plutôt passifs, ils auraient donc moins de chance de bien intégrer la matière, et surtout, de retenir ce qu'ils apprennent. Malgré ces lacunes, je crois que l'enseignement magistral peut être utile parce qu'il constitue un moyen rapide d'avoir accès à de nouvelles connaissances. L'objectif est donc de présenter les grandes lignes du système juridique canadien et québécois, pour ensuite aborder des notions propres au domaine des valeurs mobilières, en commençant par une introduction au système financier canadien, suivie d'une présentation des principales lois qui régissent le domaine, des organismes de réglementation, du fonctionnement de la société par actions, des divers types d'instruments financiers et du processus de placement de valeurs (voir Annexe 1).

Les exercices donnés aux étudiants peuvent être des textes à traduire, mais il peut aussi s'agir de l'analyse comparée d'un texte bilingue (une loi ou un prospectus, par exemple), du résumé d'un article journalistique de l'anglais vers le français, de la recherche d'équivalents français dans des articles de revues ou de journaux, de la construction d'un lexique bilingue sur un sujet donné, etc. (Voir annexe 2 pour un aperçu des types d'exercices possibles, les différentes sections correspondant à celles 
de l'annexe 1.) Un exercice est donné à la fin de chaque cours, et il est corrigé en groupe au cours suivant (sur trois heures de cours, une heure peut être consacrée à la correction). Ces exercices sont notés globalement, c'est-à-dire qu'un pourcentage de la note est accordé à la participation en classe, participation qui consiste pour les étudiants à donner leurs solutions ou leurs trouvailles.

D'autre part, tel que mentionné plus haut, mon objectif est double lorsque je prépare ces exercices, car ils doivent permettre aux étudiants d'apprendre à connaître la documentation dans le domaine et à résoudre des difficultés terminologiques particulières. En ce qui concerne le développement des aptitudes documentaires, on peut choisir des textes dans lesquels, par exemple, des extraits de lois sont cités, tout en s'assurant que ce sont des lois traduites dans les deux langues officielles. Les étudiants doivent donc trouver la loi, son titre en français, et repérer les articles qui vont leur permettre de traduire le texte en respectant la terminologie fédérale ou provinciale, tout selon la loi. Autre type d'exercice possible: fournir un texte à traduire dont le contenu porte sur une entreprise particulière, mais en veillant cette fois-ci à trouver une société qui possède un site Web bilingue, comme Alcan, Pétro-Canada ou Bombardier. Les étudiants ont alors pour tâche de trouver le site de l'entreprise en question ou encore les documents relatifs à cette entreprise accessibles sur SEDAR (le Système électronique de données, d'analyse et de recherche), ce qui leur permet de se familiariser avec les méthodes de recherche que de nombreux traducteurs juridiques utilisent dans le milieu professionnel.

Le second objectif visé étant l'acquisition de connaissances d'ordre terminologique, je distribue des textes de vulgarisation, de sorte que les étudiants aient à recourir à différents synonymes pour rendre un même terme. Le terme de security peut être traduit, selon le contexte, par instrument financier, valeur mobilière, titre, action ou obligation. Toutefois, pour être en mesure de bien comprendre ces nuances, et surtout pour pouvoir les appliquer dans une traduction, il faut être capable de distinguer des notions aussi simples qu'un titre d'emprunt et un titre de participation, ou encore une obligation et une action. Les exercices permettent de voir ces différents termes et, ainsi, de ne pas faire l'erreur de rendre security par action dans un document provenant de l'État fédéral, par exemple. On peut également demander aux étudiants de se constituer un mini-dossier terminologique sur un sujet donné. Je leur propose alors de consulter les bulletins de l'Ordre des comptables agréés du Québec (Terminologie comptable), maintenant accessibles sur Internet, et de s'inspirer de la méthodologie utilisée: «De façon générale, les articles des bulletins fournissent une description des notions traitées, précisent la problématique - laquelle découle le plus souvent d'un contexte de traduction -, font le point sur les divers choix et justifient la solution retenue» (Guével 1990: 157).

Enfin, pour ce qui est des travaux pratiques demandés aux étudiants, il s'agit de la traduction d'un texte, mais toujours accompagnée d'une bibliographie et d'un lexique bilingue comprenant le terme anglais et son ou ses équivalents français, une source et, si nécessaire, une note explicative. Cette démarche permet à la fois de développer des compétences en recherche et de mieux comprendre le sens du texte original. Par exemple, le terme insider trading comporte deux équivalents en français qui ne sont pas des synonymes: opération d'initié et délit d'initié. Lorsque les étudiants traduisent un texte où apparaît ce terme, ils constatent, en consignant les équivalents trouvés, qu'une notion importante distingue les équivalents français, la notion de 
légalité (l'opération d'initié étant permise, alors que le délit d'initié est une opération frauduleuse). La construction du lexique permet donc aux étudiants d'améliorer leur compréhension du texte en particulier et du domaine en général. Ainsi, ils ne traduisent pas «à l'aveuglette», sans comprendre l'énoncé de départ.

Un autre objectif se dissimule derrière cette obligation de produire un lexique bilingue, celui d'acquérir une démarche méthodologique qui leur permettra, dans l'avenir (et dans le présent!), de gagner du temps, car ils n'auront pas à faire deux fois la même recherche documentaire pour un terme. En outre, s'ils acquièrent cet «automatisme» de se constituer un lexique-maison chaque fois qu'ils auront un texte à traduire, ils se bâtiront (comme le font les traducteurs professionnels, d'ailleurs) une base de données personnelle qui leur permettra d'être plus efficaces, mais aussi de mieux intégrer de nouveaux concepts, deux atouts non négligeables lorsqu'ils se dirigeront vers le marché du travail.

\section{Conclusion}

J'aimerais revenir sur ce lien que je tente t'établir dans mes cours avec le marché professionnel de la traduction juridique. Il importe surtout de préciser les limites d'un tel programme. En effet, il ne suffit pas de donner des textes à traduire aux étudiants, même sous la forme d'exercices en temps limité, pour qu'ils puissent se représenter la "vraie vie» d'un traducteur professionnel. Le but, derrière cette démarche, est surtout de donner aux étudiants un avant-goût de ce qui se fait dans la profession afin qu'ils puissent décider si ce domaine est véritablement celui qui les intéresse. Car il a été fait mention des différents objectifs d'apprentissage visés dans un cours de traduction juridique: notionnel et linguistique, aptitudes documentaires, connaissances terminologiques, etc. Cependant, un objectif important a été laissé de côté: le goût de faire de la traduction juridique. Comment donner aux étudiants le goût de se diriger vers ce domaine de spécialité? Il n'y a pas vraiment de moyens précis ni de recette magique. On peut toutefois procéder à l'envers, et tenter de ne pas les décourager d'opter pour cette discipline. Pour ce faire, je crois qu'il importe de ne pas trop entrer dans les détails et complexités du domaine à l'étude, et d'en présenter plutôt les principales notions afin que les étudiants aient une vue d'ensemble qui leur permette non pas de tout savoir, mais de tout comprendre. Lorsque l'on comprend bien un domaine, on peut commencer à l'apprécier, car les angoisses et les doutes du début, liés à la peur de l'inconnu, cèdent la place au plaisir de la connaissance. Si cet objectif est atteint, en d'autres mots, si les étudiants découvrent qu'ils aiment le domaine, ils vont de leur propre chef trouver des moyens de se spécialiser davantage, en suivant, par exemple, un cours de droit des valeurs mobilières, de droit des sociétés ou de comptabilité, ou encore en faisant un stage dans le domaine, de sorte que les objectifs d'apprentissage décrits plus haut seront, par la force des choses, atteints également.

\section{RÉFÉRENCES}

Archambault, J.-P. et M.-A. Roy (1995): Initiation au droit des affaires, $2^{\text {e }}$ édition, Laval, Éditions Études Vivantes.

Cormier, M. C. (1990): «Traduction de textes de vulgarisation et de textes didactiques: approche pédagogique», Meta, 35-4, p. 676-688. 
Delisle, J. (1988): «L'initiation à la traduction économique», Meta, 33-2, p. 204-215.

Gémar, J.-C. et V. Ho-Thuy (1997): Difficultés du langage du droit au Canada, $2^{e}$ édition, Cowansville (Québec), Yvon Blais.

GÉmar, J.-C. (1988) : «La traduction juridique: art ou technique d'interprétation? », Meta, 33-2, p. 305-319.

Guével, Z. (1990) : «Traduction et développement de la terminologie française dans le domaine des affaires », Meta, 35-1, p. 154-161.

Lacasse, N. (1998): Droit de l'entreprise, $3^{\mathrm{e}}$ édition, Sillery, Les éditions Narval.

Mareschal, G. (1988) : «Le rôle de la terminologie et de la documentation dans l'enseignement de la traduction spécialisée», Meta, 33-2, p. 258-266.

Martel, M. et P. Martel (1997): La compagnie au Québec. Volume 1: Les aspects juridiques, Montréal, Wilson \& Lafleur.

Pelage, J. (1999): «La formation des traducteurs juridiques», dans Dossier: la traduction juridique et assermentée (Juillet 1999), Association des anciens élèves de l'École supérieure d'interprètes et de traducteurs de l'Université de Paris, 3 pages. Adresse URL: <http://www. geocities.com/aaeesit/juri4.html> (page consultée le 8 mai 2002).

Reed, D. G. (1993): «Some Terminological Problems of Translating Common Law Concepts from English to French», in H. B. Sonneveld et K. L. Loening (dir.), Terminology. Applications in Interdisciplinary Communication, Amsterdam/Philadelphia, John Benjamins, p. 79-86.

Reed, David G. (1979) : «Problèmes de la traduction juridique au Québec », Meta, 24-1, p. 95-102.

Roberts, R. P. (1987): «Legal Translator and Legal Interpreter Training in Canada», L'Actualité terminologique/Terminology Update, 20-6, p. 8-10.

SchWAв, W. (1984): Les anglicismes dans le droit positif québécois, Québec, Éditeur officiel du Québec.

Smart, G. (1999): «Le droit, une affaire d'inteprétation des mots», in Dossier: la traduction juridique et assermentée (Juillet 1999), Association des Anciens Elèves de l'Ecole Supérieure d'Interprètes et de Traducteurs de l'Université de Paris, 2 pages. Adresse URL: <http:// www.geocities.com/aaeesit/juri1.html> (page consultée le 8 mai 2002).

Sparer, M. (2002) : «Peut-on faire de la traduction juridique? Comment doit-on l'enseigner?», Meta, 47-2, p. 265-278.

\section{ANNEXE 1}

\section{Contenu d'un cours de traduction juridique avancé}

0. Introduction générale: le système juridique canadien et québécois

1. Système financier canadien

1.1. Instruments financiers

1.2. Marchés

1.3. Acteurs du système

2. Législation et organismes de réglementation

2.1. Législation provinciale et fédérale sur les sociétés

2.2. Loi sur les valeurs mobilières

2.3. Commission des valeurs mobilières du Québec (CVMQ) : rôle et responsabilités

2.4. Autorités canadiennes en valeurs mobilières (ACVM)

3. Société par actions

3.1. Processus de constitution

3.2. Société ouverte et société fermée

3.3. Dirigeants et actionnaires 
4. Différents types de valeur mobilière

4.1. Titres d'emprunt

4.2. Actions

4.3. Produits dérivés

4.4. Fonds communs de placement

5. Commerce des valeurs mobilières

5.1. Processus de placement

5.2. Appel public à l'épargne

5.3. Prospectus

\section{ANNEXE 2}

\section{Exercices donnés dans un cours de traduction juridique avancé}

0. Traduction de courts textes tirés d'ouvrages de droit généraux (John E.C. Brierley et Roderick A. Macdonald, Quebec Civil Law. An Introduction to Quebec Private Law, 1993; Gerald L. Gall, The Canadian Legal System, 1990.)

1. Traduction de textes tirés d'ouvrages d'introduction au droit des sociétés et au système financier

(Anthony J. VanDuzer, The Law of Partnerships and Corporations,1997; W. T. Hunter, Canadian Financial Markets, 1988.)

2. Analyse comparée d'articles de la Loi canadienne sur les sociétés par actions; analyse comparée de textes bilingues extraits du site web des ACVM: critiques et commentaires

3. Élaboration d'un lexique bilingue des titres de fonction des dirigeants d'entreprise à partir d'un texte à traduire

4. Résumés d'articles de l'anglais vers le français (section finance du Globe and Mail, par exemple)

5. Traduction d'un prospectus (ou d'une partie seulement); analyse comparée d'un prospectus bilingue 